\title{
Gastric involvement in AIDS associated cryptosporidiosis
}

\author{
P Rossi, F Rivasi, M Codeluppi, A Catania, A Tamburrini, E Righi, E Pozio
}

\begin{abstract}
Background-Cryptosporidiosis has been shown to be a common cause of diarrhoea in both immunocompetent and immunosuppressed individuals. There are very few data on the distribution of Cryptosporidium parvum along the gastrointestinal tract.

Aims-To evaluate the location of Cryptosporidium parasites in the digestive tract of patients with AIDS.

Methods-Gastrointestinal localisation of C parvum was studied in 71 patients with AIDS who underwent upper and/or lower endoscopy with biopsy for chronic diarrhoeal illness and/or other gastrointestinal disorders of unexplained origin.

Results-Twenty four individuals (33.8\%) were positive for $C$ parvum, of which 16 $(88.9 \%)$ had parasites in the gastric epithelium. Most patients with gastric localisation of $C$ parvum did not show specific symptoms indicating the presence of this parasite in the stomach.

Conclusions-Gastric involvement in AIDS related cryptosporidiosis is more frequent than expected, but no clear correlation between gastric location and related clinical and pathological features was observed.

(Gut 1998;43:476-477)
\end{abstract}

Keywords: Cryptosporidium parvum; AIDS; gastric location

Laboratory of

Parasitology, Istituto

Superiore di Sanità,

Rome, Italy

P Rossi

A Tamburrini

E Pozio

Department of

Morphological

Sciences and Forensic

Medicine

F Rivasi

E Righi

Department of Infectious Diseases, University of Modena, Modena, Italy M Codeluppi

A Catania

Correspondence to: Dr E Pozio, Laboratory of Parasitology, Istituto Superiore di Sanità, Viale Regina Elena 299, 00161 Rome, Italy.

Accepted for publication 26 March 1998
Methods
We enrolled in this study all patients with AIDS
(as defined by the criteria of the US Centers for
Disease Control and Prevention) who under-
went gastrointestinal endoscopy with biopsy at
the Infectious Disease Clinic of the University
of Modena, in the period 1986-1996. The
cause for referral was chronic diarrhoeal illness
and/or other gastrointestinal disorders of unex-

Methods
We enrolled in this study all patients with AIDS
(as defined by the criteria of the US Centers for
Disease Control and Prevention) who under-
went gastrointestinal endoscopy with biopsy at
the Infectious Disease Clinic of the University
of Modena, in the period 1986-1996. The
cause for referral was chronic diarrhoeal illness
and/or other gastrointestinal disorders of unex-

Methods
We enrolled in this study all patients with AIDS
(as defined by the criteria of the US Centers for
Disease Control and Prevention) who under-
went gastrointestinal endoscopy with biopsy at
the Infectious Disease Clinic of the University
of Modena, in the period 1986-1996. The
cause for referral was chronic diarrhoeal illness
and/or other gastrointestinal disorders of unex-

Methods
We enrolled in this study all patients with AIDS
(as defined by the criteria of the US Centers for
Disease Control and Prevention) who under-
went gastrointestinal endoscopy with biopsy at
the Infectious Disease Clinic of the University
of Modena, in the period 1986-1996. The
cause for referral was chronic diarrhoeal illness
and/or other gastrointestinal disorders of unex- plained origin (epigastric pain, abdominal pain, vomiting, etc.), or the follow up of other pathologies. Endoscopic procedures were performed either by a gastroscope for upper endoscopy or by a colonoscope for lower endoscopy. Multiple biopsy specimens were taken from the oesophagus, stomach, duodenum, jejunum-ileum, colon, and rectum. Formalin fixed biopsy specimens were routinely processed for histological examination. Faecal samples were examined by modified acid fast staining and by immunofluorescence for $C$ parvum, after formalin-ether concentration.

\section{Results and discussion}

Seventy one patients with AIDS underwent gastrointestinal endoscopy with biopsy during the study period. Most individuals $(n=63$, $88.7 \%$ ) were young men (mean age 37 years, range 27-69), with an average of $62 \mathrm{CD} 4+$ / $\mathrm{mm}^{3}$ (range 0-499). A total of 215 biopsy specimens were examined. Some individuals underwent complete oesophagogastroduodenoscopy, while others had partial upper and/or lower endoscopy with biopsy. Twenty four individuals $(33.8 \%)$ were positive for $C$ parvum by histological examination; 22 of them (95.8\%) had multiple biopsy sites simultaneously positive for the parasite. Parasites were found in $16(88.9 \%)$ of the 18 patients with AIDS who underwent gastric biopsy, in 19 $(86.3 \%)$ undergoing duodenal biopsy, in 16 $(100 \%)$ undergoing ileo-jejunal biopsy, in 11 (78.6\%) undergoing colonic biopsy, and in six individuals $(85.7 \%)$ undergoing rectal biopsy. No parasites were found in oesophageal biopsy specimens. Oocysts were detected in stools from $12(50.0 \%)$ of the 24 patients with AIDS with positive biopsy specimens. Only three $(18.7 \%)$ patients with AIDS with gastric localisation of $C$ parvum had vomiting, and only one $(6.2 \%)$ reported epigastric pain. The results of endoscopy of the 16 patients with AIDS with gastric cryptosporidiosis showed the following: hyperaemia $(n=8)$, oedema $(n=4)$, erosions $(n=4)$, diffuse flogosis $(n=2)$, light atrophy $(n=1)$, bleeding $(n=1)$, polyps $(n=1)$, and ulcerations $(n=1)$, and no alterations in five patients. The histological evaluation of these 16 patients with AIDS showed: acute inflammatory infiltrate $(n=7$ patients), chronic inflammatory infiltrate $(n=6)$, erosive and hyperplastic regenerative process $(n=3)$, and no alterations in five patients. Parasites were found mostly in the antrum and had a patchy distribution, with parasitised glands being contiguous to negative ones; their presence was related to the degree of alteration of the gastric mucosa, as areas showing 


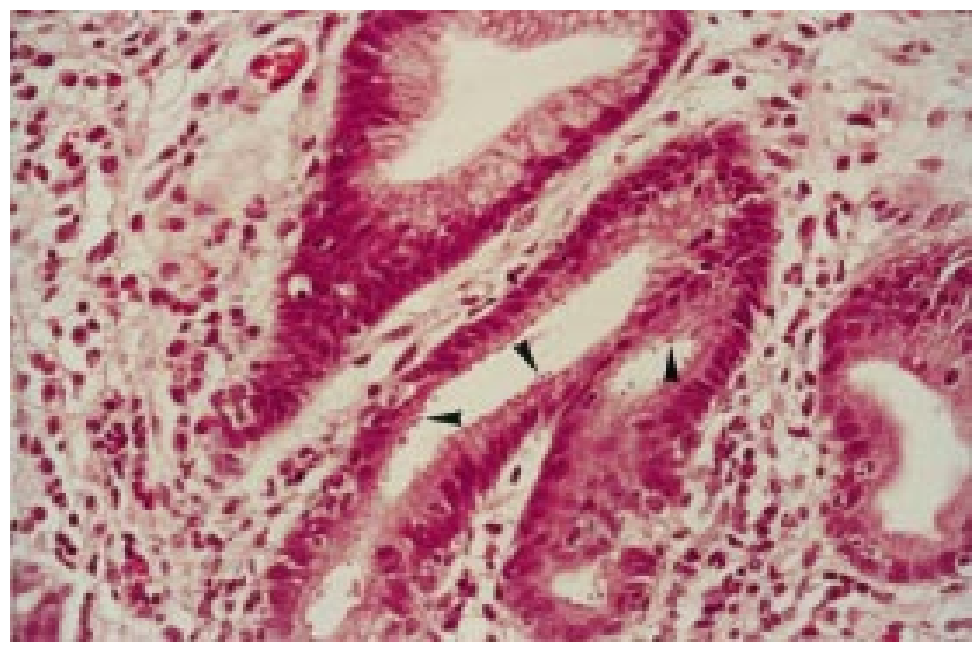

Figure 1 Patchy distribution of Cryptosporidium parasites (arrowheads) in the gastric mucosa (antrum): a parasitised gland is contiguous to free ones (haematoxylin and eosin, original magnification $\times 200)$.

abnormal architecture were more densely colonised by parasites (fig 1 ).

The frequency of gastric involvement in cryptosporidiosis was higher than expected. A recent review of the literature reported a total of 16 cases of gastric cryptosporidiosis ${ }^{3}$; six additional cases were also documented by endoscopic examination, ${ }^{4-7}$ and three cases at autoptic evaluation. ${ }^{8}$ It has been suggested that colonisation of gastric mucosa takes place in a retrograde manner from the small bowel to the stomach, where the parasite may develop depending on favourable conditions due to concomitant pathologies, such as hypochlorhydria, which is present in more than one third of patients with AIDS. ${ }^{2}$ Gastric localisation of $C$ parvum in patients with AIDS has been reported to be associated with gastritis and antral narrowing. Our results show that gastric cryptosporidiosis is not always related to a specific symptomatology, as patients with a large number of parasites in the stomach were asymptomatic. In one third of patients with AIDS, no evident alterations of the gastric epithelium were associated with the presence of parasites, whereas there was a relation between the degree of mucosal alteration and the number of parasites colonising gastric glands. However, it is still unclear whether these alterations are caused by the parasites. In conclusion, the lack of a pathognomonic clinical picture and of an endoscopic appearance related to gastric cryptosporidiosis suggests that upper endoscopy with randomly taken multiple biopsy specimens should be adopted for immunodepressed patients with AIDS, to avoid any underestimation of gastric location.

Financial support was provided by the Istituto Superiore di Sanità, Ministero della Sanità, National AIDS Project, contract $10 \mathrm{~A} / \mathrm{K}$

1 Goodgame RW, Genta RM, White AC, et al. Intensity of infection in AIDS-associated cryptosporidiosis. F Infect Dis 1993;167:704-9.

2 Kotler DP. Gastrointestinal manifestations of human immunodeficiency virus infection. Adv Intern Med 1995; 40:197-242.

3 Ventura G, Cauda R, Larocca LM, et al. Gastric cryptosporidiosis complicating HIV infection: case report and review of the literature. Eur $\mathcal{F}$ Gastroenterol Hepatol 1997;9:307-10

4 Jautzke G, Niedobitek F, Arasteh K, et al. Kryptosporidien in Biopsien aus dem Magen-Darm Trakt. Pathologie 1986; 7:302-3.

5 Zambrano Nuñez MR, Sakai P, Ishioka S, et al. Gastroduodenite erosiva associada a Cryptosporidium num paciente com sindrome de imunodeficiencia adquirida. Rev Hosp Clin Fac Med S Paulo 1990;45:188-9.

6 Besasso HR, Macías J, Trombetta LA, et al. Gastritis erosiva asociada a Cryptosporidium en dos pacientes con SIDA. asociada a Cryptosporidium en dos pacient

7 Greenberg PD, Koch J, Cello JP. Diagnosis of Cryptosporidium parvum in patients with severe diarrhea and osporidium parvum in patients with
AIDS. Dig Dis Sci 1996;41:2286-90.

8 Godwin TA. Cryptosporidiosis in the acquired immunodeficiency syndrome: a study of 15 autopsy cases. Hum Pathol 1991;22:1215-24 